\title{
Diamond Sensors in HEP
}

\section{CERN RD-42 Collaboration}

M.Mikuž $^{1,12}$, M.Artuso ${ }^{24}$, F.Bachmair ${ }^{28}$, L.Bäni ${ }^{28}$, V.Bellini $^{2}$, V Belyaev ${ }^{15}$, E.Berdermann $^{8}$, J-M.Brom ${ }^{10}$, M.Bruzzi ${ }^{5}$, B. Caylar ${ }^{14}$, M.Červ ${ }^{3}$, G.Chiodini ${ }^{31}$, D.Chren ${ }^{22}$, V.Cindro ${ }^{12}$, G.Claus ${ }^{10}$, M.Cristinziani $^{1}$, S Costa $^{2}$, J.Cumalat $^{23}$, R.D'Alessandro ${ }^{6}$, W.de Boer ${ }^{13}$, D.Dobos ${ }^{3}$, W.Dulinski ${ }^{10}$, V.Eremin ${ }^{9}$, R.Eusebi ${ }^{29}$, H.Frais-Kölbl ${ }^{4}$, C.Gallrapp ${ }^{3}$, K.K.Gan ${ }^{16}$, J.Garofoli ${ }^{24}$, M.Gastal ${ }^{3}$, M.Goffe ${ }^{10}$, J.Goldstein $^{20}$, A.Golubev ${ }^{11}$, L.Gonella ${ }^{1}$, A.Gorišek ${ }^{12}$, E.Grigoriev ${ }^{11}$, J.GrosseKnetter $^{27}$, M.Guthoff ${ }^{13}$, I.Haughton ${ }^{26}$, D.Hidas ${ }^{17}$, D.Hits ${ }^{28}$, M.Hoeferkamp ${ }^{25}$, J.Hosselt $^{10}$, F.Hügging ${ }^{1}$, H.Jansen ${ }^{3}$, J.Janssen ${ }^{1}$, H.Kagan ${ }^{16}$, R.Kass ${ }^{16}$, G.Kramberger $^{12}$, S.Kuleshov ${ }^{11}$, S.Kwan ${ }^{7}$, S.Lagomarsino ${ }^{6}$, A.Lo Giudice ${ }^{18}$, C.Maazouzi $^{10}$, I.Mandić ${ }^{12}$, C.Manfredotti $^{18}$, C.Manfredotti $^{18}$, A.Martemyanov ${ }^{11}$, C.Mathieu10, H.Merritt ${ }^{16}$, M.Moench ${ }^{28}$, R.Mori ${ }^{5}$, J.Moss ${ }^{16}$, R.Mountain ${ }^{24}$, G.Oakham ${ }^{21}$, T.Obermann ${ }^{1}$, A.Oh ${ }^{26}$, P.Olivero ${ }^{18}$, G.Parrini ${ }^{6}$, H Pernegger ${ }^{3}$, R.Perrino ${ }^{31}$, M.Pomorski ${ }^{14}$, R.Potenza ${ }^{2}$, A.Quadt ${ }^{27}$, S.Roe ${ }^{3}$, S.Schnetzer ${ }^{17}$, T.Schreiner ${ }^{4}$, S.Saidel ${ }^{25}$, A.Sfyrla ${ }^{3}$, S.Sciortino ${ }^{6}$, S.Smith ${ }^{16}$, B.Sopko ${ }^{22}$, S.Spagnolo ${ }^{31}$, S.Spanier ${ }^{30}$, K.Stenson ${ }^{23}$, R.Stone ${ }^{17}$, C.Sutera ${ }^{2}$, W.Trischuk ${ }^{19}$, C.Tuve ${ }^{2}$, V.Tyzhnevyi ${ }^{26}$, L.Upleger ${ }^{7}$, J Velthuis ${ }^{21}$, N.Venturi $^{19}$, E.Vittone $^{18}$, S.Wagner ${ }^{23}$, R.Wallny ${ }^{28}$, J.C.Wang ${ }^{24}$, R.Wang ${ }^{25}$, P.Weilhammer ${ }^{3}$, J.Weingarten ${ }^{27}$, C.Weiss $^{3}$, T.Wengler ${ }^{3}$, N.Wermes ${ }^{1}$, M.Zavrtanik ${ }^{12}$

${ }^{1}$ Universität Bonn, Bonn, Germany, ${ }^{2}$ INFN/University of Catania, Catania, Italy, ${ }^{3}$ CERN, Geneva, Switzerland, ${ }^{4}$ Fachhochschule fur Wirtschaft und Technik, Wiener Neustadt, Austria, ${ }^{5}$ INFN/University of Florence, Florence, Italy, ${ }^{6}$ Department of Energetics/INFN, Florence, Italy, ${ }^{7}$ FNAL, Batavia, USA, ${ }^{9}$ Ioffe Institute, St. Petersburg, Russia, ${ }^{10}$ IPHC, Strasbourg, France, ${ }^{11}$ ITEP, Moscow, Russia, ${ }^{12}$ Jožef Stefan Institute and Physics Department, University of Ljubljana, Ljubljana, Slovenia, ${ }^{13}$ Universität Karlsruhe, Karlsruhe, Germany, ${ }^{14}$ CEA/LIST Technologies Avancées, Saclay, France ${ }^{15}$ MEPHI Institute, Moscow, Russia, ${ }^{16}$ The Ohio State University, Columbus, OH, USA, ${ }^{17}$ Rutgers University, Piscataway, NJ, USA, ${ }^{18}$ University of Torino, Torino, Italy, ${ }^{19}$ University of Toronto, Toronto, Canada, ${ }^{20}$ University of Bristol, Bristol, UK, ${ }^{21}$ Carleton University, Ottawa, Canada, ${ }^{22}$ Czech Technical University, Prague, Czech Republic, ${ }^{23}$ University of Colorado, Boulder, CO, USA, ${ }^{24}$ Syracuse University, Syracuse, NY, USA, ${ }^{25}$ University of New Mexico, Albuquerque, NM, USA, ${ }^{26}$ University of Manchester, Manchester, UK, ${ }^{27}$ Universität Göttingen, Göttingen, Germany, ${ }^{28}$ ETH Zürich, Zürich, Switzerland, ${ }^{29}$ Texas A\&M College, Park Station, TX, USA, ${ }^{30}$ University of Tennessee, Knoxville, TN, USA, ${ }^{31}$ INFN-Lecce, Lece, Italy.

${ }^{1}$ Speaker, E-mail: Marko.Mikuz@ijs.si 


\section{ABSTRACT}

With the first three years of the LHC running well underway, and luminosity upgrades expected towards the end of the decade, ATLAS and CMS are planning to upgrade their innermost tracking layers with the utmost radiation hard technologies. Chemical Vapour Deposition (CVD) diamond has been used extensively in beam conditions monitors as the innermost detectors in the highest radiation areas of BaBar, Belle, CDF and all LHC experiments. This material is now being considered as a sensor material for the innermost layer(s) of the upgraded trackers. Recently the CERN RD42 collaboration constructed, irradiated and tested polycrystalline and single-crystal CVD diamond sensors to the highest fluences expected at the innermost tracking layers of the HL-LHC. We present beam test results of chemical vapour deposition diamond to fluences in excess of $10^{16}$ protons $/ \mathrm{cm}^{2}$ illustrating that both polycrystalline and single-crystal chemical vapour deposition diamonds follow a single damage curve. We briefly describe the performance of the diamond-based ATLAS beam monitoring devices and discuss plans for their upgrade to a diamond tracker during the 2013/14 LHC consolidation shut-down.

36th International Conference on High Energy Physics

July 4-11, 2012

Melbourne, Australia 


\section{Introduction}

Progress in experimental particle physics in the coming decade depends crucially upon the ability to carry out experiments at high energies and high luminosities. These conditions imply that future experiments will take place in extremely harsh radiation areas, with hadron fluences surpassing $10^{16} / \mathrm{cm}^{2}$ for innermost tracking layers or even $10^{17}$ in forward calorimeters. In order to perform these complex and expensive experiments new radiation hard technologies are being developed. Chemical Vapour Deposition (CVD) diamond is being pursued by the CERN RD-42 collaboration [1] as a radiation tolerant material for use very close to the interaction region. During the past few years many CVD diamond devices have been manufactured and tested. As a detector for high radiation environments CVD diamond benefits substantially from its intrinsic radiation hardness (large displacement energy $42 \mathrm{eV} /$ atom), very low leakage current, low dielectric constant, fast signal collection and ability to operate at room temperature. As a result CVD diamond now has been used extensively in beam conditions monitors as the innermost detectors in the highest radiation areas of colliders. CVD diamond is being considered as a sensor material for the future particle tracking detectors closest to the interaction region where the most extreme radiation conditions exist. We present the state-of-the-art of the radiation tolerance of the highest quality CVD diamond material for a range of proton energies, pions and neutrons obtained from strip detectors constructed with this material.

Recently single crystal CVD diamond material has been developed which resolves many of the issues associated with polycrystalline material. We also present recent results on radiation tolerance obtained from strip detectors constructed from this new diamond material. We discuss the use of diamond detectors and their survivability in the highest radiation environments.

Currently diamond is deployed in HEP experiments for two tasks: for beam conditions monitoring and measurement of luminosity. Beam conditions can be deduced either from beam induced current measurements replacing the commonly used ionization chambers by CVD diamond pad detectors (e.g. ATLAS BLM; CMS BCM1, BCM2, LHCb, ALICE) or by particle counting (ATLAS BCM; CMS BCMF). Due to its fast response and insensitivity to pile-up, ATLAS BCM also serves as the preferred luminosity monitor. As a result of positive experience with the ATLAS BCM an upgraded Diamond Beam Monitor (DBM) is being prepared for installation during the 2013-14 LHC shutdown. This detector will be a true diamond sensor based tracker, consisting of 8 three-layer telescopes of pixelated diamond detectors. Each plane will have $\sim 27 \mathrm{k}$ active pixels. The telescopes will provide sub-mm impact parameter resolution adding spatial information on the origin of backgrounds to the already precise (sub-ns) timing information from the BCM.

\section{Diamonds as Particle Detectors}

Diamond, because of its large resistivity, can be operated as a solid-state ionization chamber. In Fig. 1, the basic principle of using diamond as a particle detector is shown [2]. A voltage is applied across a layer of diamond a few hundred microns thick. When a charged particle traverses the diamond, atoms in crystal lattice sites are ionized, promoting electrons into the conduction band and leaving holes in the valence band. On average, 36 electron-hole pairs 
are created in each $\mu \mathrm{m}$ of diamond traversed by a minimum ionizing particle. These charges drift across the diamond in response to the applied electric field producing a detectable electric signal. Since there may be traps in CVD material we often use the charge collection distance $(C C D)$ to characterize the material. $C C D$ corresponds to the average distance the electron-hole pairs move apart. In thin diamond $C C D$ is limited by sensor dimension, while in thick (thickness > $C C D$ ) diamond $C C D$ approaches the sum of mean free paths of electrons and holes.

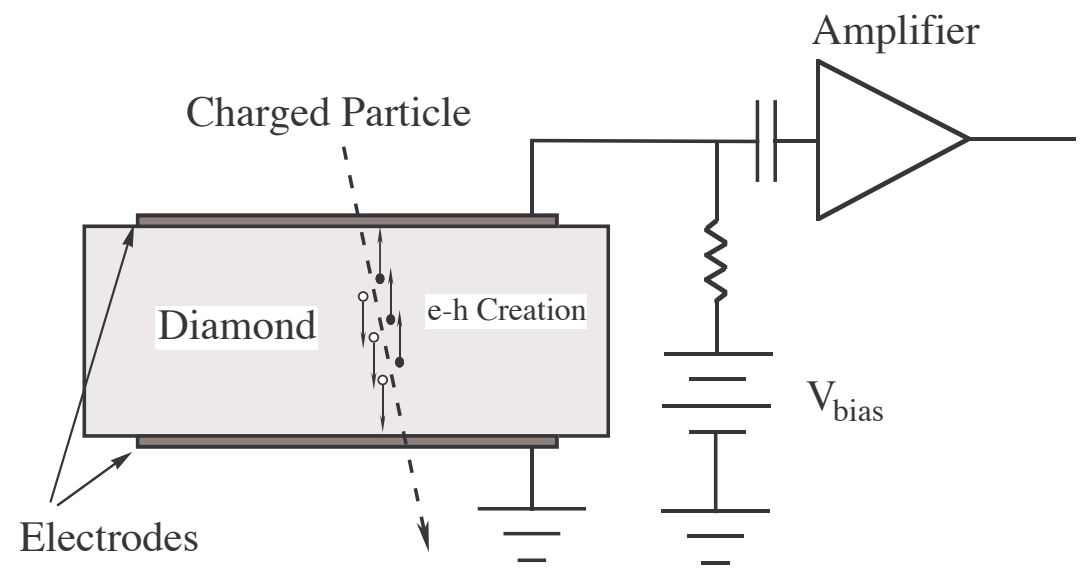

Figure 1: Schematic view of operation of a diamond particle detector. The metal electrodes can be segmented to produce a pixel or strip detector.

There are two flavours of CVD diamond: polycrystalline (pCVD) and single crystal ( $\mathrm{scCVD}$ ). The scCVD is grown on a high-pressure high-temperature diamond substrate and forms a perfect diamond lattice. On the other hand, pCVD is grown on a non-diamond substrate, therefore small crystal grains in random orientations start forming on the substrate. The grains grow, and the larger ones have the tendency to grow faster, terminating the growth of the smaller ones. Therefore the average grain size increases across the pCVD thickness from the substrate to the growth side. Also the charge collection properties of the material exhibit improvement with growth thickness.

While scCVD exhibits very little trapping, and therefore its $C C D$ matches the detector thickness, $C C D$ of pCVD's is limited by trapping. It is helpful to grow thick pCVD wafers, with thickness in excess of $1 \mathrm{~mm}$, and polish off the substrate side, keeping the high quality material with large grains. Top quality pCVD material today can be grown in 6" wafers, and can exhibit $C C D$ close to $300 \mu \mathrm{m}$ at $500 \mu \mathrm{m}$ thickness and electric field of $2 \mathrm{~V} / \mu \mathrm{m}$. The superb quality of scCVD is for the time heavily offset by size and price; scCVD, in contrast to 6" pCVD wafers, is limited to sizes of $1 \times 1 \mathrm{~cm}^{2}$, and the price difference for the small pieces available is nearly an order of magnitude.

\section{Diamond Sensor Suppliers}

Traditionally all supplies of electronic grade diamond for HEP applications came from a single manufacturer [3] despite all efforts of CERN RD-42 to establish additional suppliers and foster competition, especially in view of a potentially sizeable demand of sensors for a HL-LHC tracking layer. Established with the aim of marketing electronic grade diamond the producer ran 
into commercial difficulties to the point in May 2012 when the owners decided not to sustain operation. Partly its mission is maintained by one of the former owners who are offering the same product line under their own brand name [4]. In 2012 a newly grown 5" pCVD wafer was obtained for testing (Fig.2a). Ten regions of $\sim 2 \mathrm{~cm} \times 2 \mathrm{~cm}$ were selected on this wafer and the 10 diamond sensors were delivered as part of the ATLAS Diamond Beam Monitor (DBM) project.

Following successful trial results, an additional manufacturer [5], regularly producing optical grade diamond, offered sensors for the ATLAS DBM. A wafer of 6" size was grown to honour an order of ten $2 \mathrm{~cm} \times 2 \mathrm{~cm}$ sensors (Fig. 2b).

Figure 2: Recent $p C V D$ diamond wafers delivered: 2a(left): Wafer from [4], 5" diameter, with test-dots on a $1 \mathrm{~cm}$ grid. Markings delimit cut-outs for ten DBM sensors. 2b(right): 6 " wafer from [5] with the equivalent test-dot pattern.
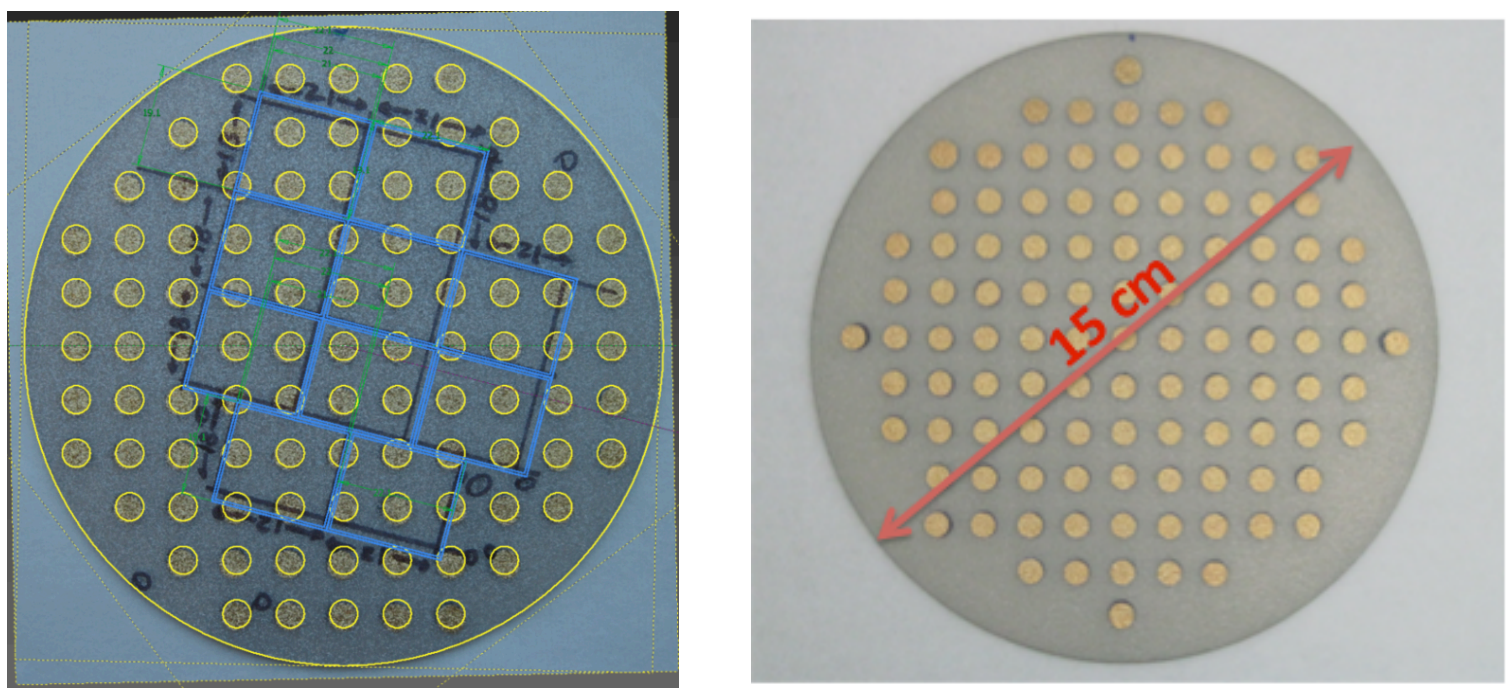

\section{Radiation Damage}

Diamond, because of its intrinsically large displacement energy of $42 \mathrm{eV}$ per atom $(26 \mathrm{eV}$ for $\mathrm{Si}$ ), is believed to exhibit favourable radiation resistance. In contrast to silicon, radiationinduced levels exhibit no noticeable effect on the leakage current, as even if located close to mid-gap $\left(E_{t} \sim 2.5 \mathrm{eV}\right)$ they can't act as generation centres at room temperature. They can, however, as in radiation-damaged silicon, trap drifting charge. If sufficiently separated from the conduction band (deep traps) the de-trapping times are large, even of order of months, so the charge is lost for the signal. Usually an occupied trap cannot trap the same charge carrier, so it remains passivized for the de-trapping time. It can, however, trap the carrier of the opposite charge, effectively serving as a recombination centre. The cross sections for the capture of the two carrier species (electrons and holes) can differ by orders of magnitude, with the larger cross section exhibited by a charged (empty) trap than the neutral (filled) one. This forms the fundament of the "pumping" procedure, where diamonds are exposed to ionizing radiation, which fills the traps and passivizes them. Pumping is done before the measurement using a strong ${ }^{90} \mathrm{Sr}$ source. It is inherently present in hadron colliders where tracking detectors are exposed to charged particle fluxes in excess of $\mathrm{MHz} / \mathrm{cm}^{2}$. 
Pumping could in principle affect the space charge and thus the electric field distribution in the sensor bulk. As no strong effects are observed one could conclude that the trapping of electrons and holes is of similar probability, thus the resulting space charge largely cancels out.

Traps can be present already in non-irradiated diamond because of lattice imperfections or be radiation-induced as a result of atom displacements. The former should be more abundant in the less perfect pCVD diamond, where in addition charge can also be trapped on grain boundaries. The introduction traps by radiation can be thought as non-discriminating between the two CVD diamond flavours, pCVD and scCVD, and the two trapping sources should be additive. As the mean free path $(m f p)$ of each carrier is inversely proportional to the relevant trap density, the resulting model is written as:

$$
1 / m f p_{e, h}=1 / m f p_{0 ; e, h}+k_{m f p ; e, h} \times \Phi,
$$

with $k_{m f p}$ the radiation damage constant and $m f p_{0}$ the value of $m f p$ before irradiation.

In thick detectors $C C D$, being equal to the sum of $m f p_{e, h}$, is observed to follow the sum of two hyperbolae given by Eq. (1), which add up to a single hyperbola with $1 / m f p_{0}=1 / m f p_{0, e}+$ $1 / m f p_{0, h}$ and $k_{m f p}=k_{m f p, e}+k_{m f p, h}$. Empirically, measuring $C C D$ with strip detectors, the collected signal is quasi independent on the electric field direction, indicating that $m f p$ of electrons roughly matches that of the holes $\left(m f p_{e} \sim m f p_{h}\right)$.

For thinner detectors the detector dimension limits the measured $C C D$. As the extreme case, non-irradiated scCVD exhibits an essentially infinite $m f_{0}$, thus there $C C D$ equals the detector thickness. For intermediate thicknesses the correspondence between $C C D$ and $m f p=$ $m f p_{e}+m f p_{h}$ in the assumption $m f p_{e} \sim m f p_{h}$ is given by:

$$
C C D=2 \times m f p\left[1-\frac{m f p}{t}\left(1-\exp \left(-\frac{t}{m f p}\right)\right)\right],
$$

where $t$ is the detector thickness.

Ideally, to test for radiation hardness, detectors should be irradiated with the particle spectrum encountered at the targeted application. For LHC and its upgrades the prevalent species close to the interaction region are pions, their spectrum peaked at $\sim 2 \mathrm{GeV}$, with FWHM from $300 \mathrm{MeV}$ to $6 \mathrm{GeV}$ [6]. Such irradiation facilities do not exist, at least not in the sought fluence range. Therefore damage modelling is needed. Often the damage is taken proportional to non-ionizing energy loss (NIEL) of the respective particle. The NIEL assumption proved useful for silicon, although also there departures from predictions have been observed. With the sources available, it sounds plausible to concentrate on charged hadrons of similar energy, and then do the residual scaling with interpolation. Luckily enough, the CERN PS irradiation facility with $24 \mathrm{GeV}$ protons and the LANL LANSCE facility with $800 \mathrm{MeV}$ protons bridge the relevant energy region. Models predict damage variation of factor 2 (NIEL based - [7]) or even only $20 \%$ (displacement per atom (DPA) based - [8]) across this energy interval.

Four diamond sensors have been irradiated at each facility, 2 pCVD and 2 scCVD at CERN, and $3 \mathrm{pCVD}$ and $1 \mathrm{scCVD}$ at Los Alamos. The administrated fluence spans the range relevant for the HL-LHC upgrade. $C C D$ was obtained before irradiation and after each irradiation step by turning the sensor into a strip detector and measuring its response to the CERN SPS high-energy pion test beam. For each of the sensors, fluence dependence was fitted 
with the linearized form of Eq. 1 (1/mfp vs. fluence - Figs. 3). Eq. 2 was used to obtain $m f p$ from the measured $C C D$, with $m f p_{0}$ either deduced from measured $C C D$ before irradiation or taken as infinite for the scCVD samples. Errors on $C C D$ and fluence were obtained from the measurement or given by the irradiation facilities, respectively, and are typically about $10 \%$ of the respective values. A linear fit with $k_{m f p}$ and $m f p_{0}$ as the free parameters was performed, where the fitted $m f p_{0}$ is relevant only for samples with more than one irradiation step.
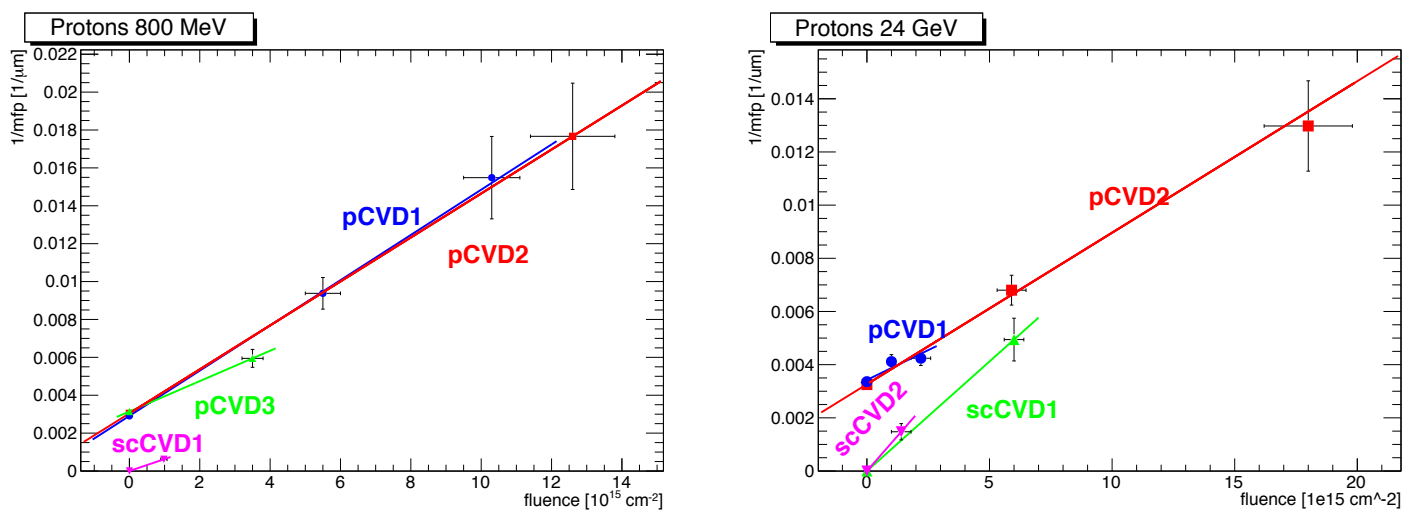

Figure 3: Dependence of 1/mfp vs. fluence for irradiations with $800 \mathrm{MeV}$ protons at LANL (left) and 24 GeV protons at CERN. Each of the four diamonds is fitted with a linear model according to Eq. 2.

The resulting $k_{m f p}$ for all the samples are depicted in Figs. 4. They appear consistent among each other, indicating a common mechanism of radiation damage. The consistency in averaging the samples is manifested better on the $24 \mathrm{GeV}$ data, although also the average $\chi^{2}$ for the 800 $\mathrm{MeV}$ data amounts to an acceptable 7.3 for 3 degrees of freedom. The resulting average $k_{m f p}$ are $(0.62 \pm 0.07) \times 10^{-18} \mu \mathrm{m}^{-1} \mathrm{~cm}^{-2}$ for $24 \mathrm{GeV}$ and $(0.95 \pm 0.09) \times 10^{-18} \mu \mathrm{m}^{-1} \mathrm{~cm}^{-2}$ for $800 \mathrm{MeV}$ protons. The damage ratio seems to favour the DPA prediction, but one should treat these results as preliminary: data and their errors are still being scrutinized, and there is more data to be added to the analysis.
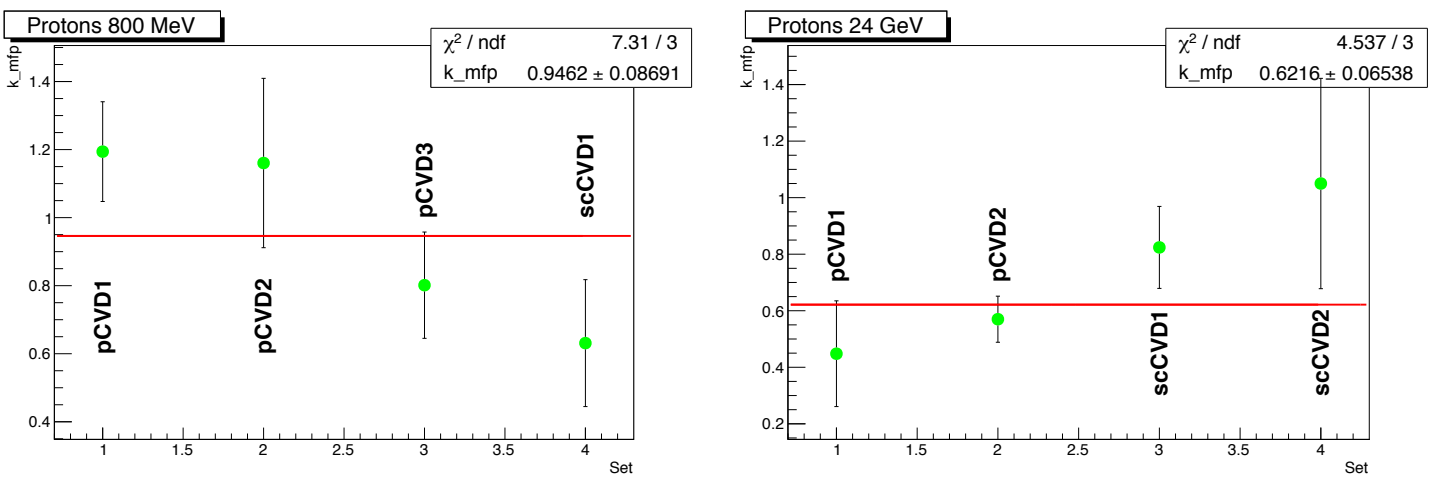

Figure 4: Consistency of the four $k_{m f p}$ at each proton energy obtained from the fit in Fig. 3: $800 \mathrm{MeV}$ protons (left) and $24 \mathrm{GeV}$ protons (right). The line denotes a constant fit to the four values, the fit result and $\chi^{2}$ indicated in the figures

Having obtained average $k_{m f p}$ for each data set it is possible to exhibit consistency of various samples with Eq. 1 by using $k_{m f p}$ to turn $m f p_{0}$ into a fluence offset $\Phi_{0}=\left(k_{m f p} m f p_{0}\right)^{-1}$. This 
offset is of order of $3-5 \times 10^{15} \mathrm{~cm}^{-2}$ for pCVD of today's state-of-the-art quality, and can be regarded as the fluence headroom if one were able to use ultimate quality (scCVD) diamond instead. The resulting plots are shown in Figs. 5 with one-sigma bands indicated. All results conform to Eq. 1 to better than 2 sigma, demonstrating validity of the assumption of a common radiation damage mechanism in CVD diamond.
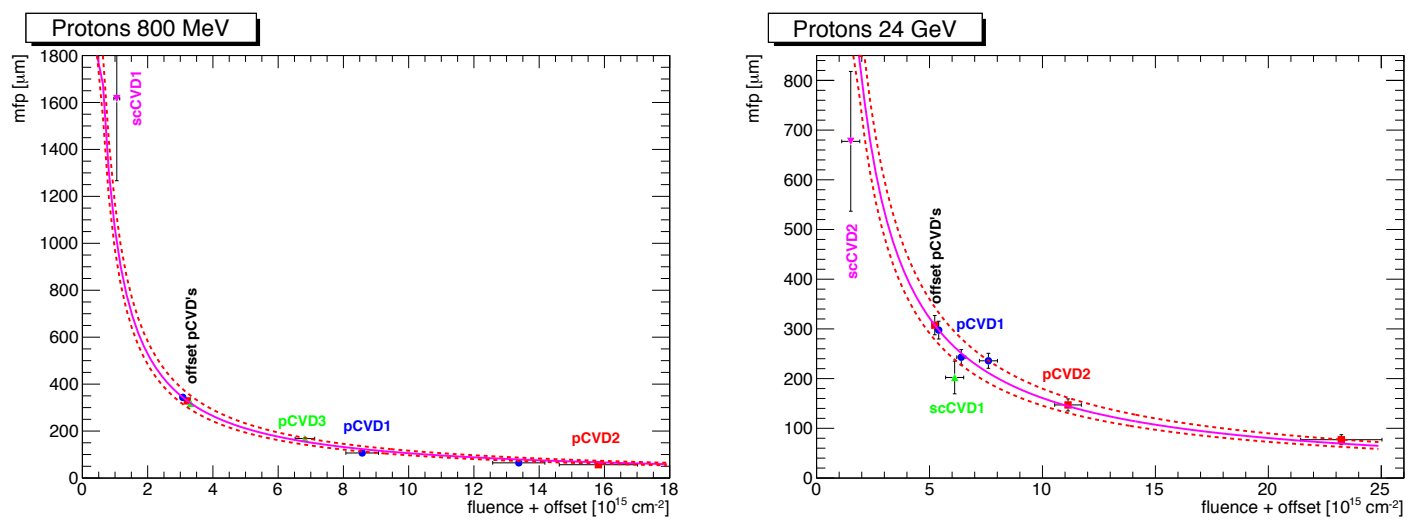

Figure 5: Plot of all irradiation data: $800 \mathrm{MeV}$ protons (left) and $24 \mathrm{GeV}$ protons (right). The line denotes Eq. 2 with the average $k_{m f p}$ and infinite initial $m f p$. The $p C V D$ diamond points have been offset as discussed in the text. The dotted line represents variation of $k_{m f p}$ by one standard deviation.

Neutrons constitute a minor part of order of $10 \%$ of the fluence at the innermost tracking layer, their spectrum ranging between $1 \mathrm{MeV}$ and $5 \mathrm{GeV}$. Preliminary results of a study [9] with reactor neutrons $(\sim 1 \mathrm{MeV})$ indicate damage a factor of $6 \pm 1$ larger than those of $24 \mathrm{GeV}$ protons. The same diamonds measured in the CERN SPS test-beam exhibit about $50 \%$ larger charge collection at the highest fluence than measured with the ${ }^{90} \mathrm{Sr}$ source in [9], indicating a relative factor of 4 for the neutron damage. These results match rather well the DPA prediction [8], while NIEL predicts only $40 \%$ larger damage, albeit for both predictions the comparison was taken at the lowest given point at $10 \mathrm{MeV}$ and not around $1 \mathrm{MeV}$. Using DPA scaling and averaging over the neutron spectrum, neutrons should contribute about $20 \%$ to the total radiation damage or a quarter of that of pions. Thus the final prediction of charge from a pCVD $\left(C C D_{0}=200 \mu \mathrm{m}\right)$ diamond detector after $10^{16} \mathrm{~cm}^{-2}$ of hadron fluence at HL-LHC is about 2000 $\mathrm{e}_{0}$, with large and hard to be estimated uncertainties mainly resulting from the non-established scaling of proton damage to pions, and scaling of neutron damage across the spectrum.

\section{ATLAS Beam Monitoring}

Currently diamond sensors have found application as beam monitoring devices in all LHC detectors. ATLAS and CMS use diamond detectors both in particle counting (ATLAS BCM; CMS BCMF) and current integration mode (ATLAS BLM; CMS BCM1, BCM2). Both experiments plan an upgrade with diamond tracking telescopes, ATLAS with the DBM project and CMS with the Pixel Luminosity Telescope (PLT) project. The CMS activities being covered in [10], in further discussion we focus on ATLAS.

ATLAS BCM is described in detail in [11]. In eight modules, 4 per side, pairs of back-toback $1 \mathrm{~cm} \times 1 \mathrm{~cm}$ pCVD diamond sensors are deployed. Read-out by fast electronics enables superb timing resolution with TOF between two modules measured at better than $1 \mathrm{~ns}$. Based on 
TOF signature beam-background events can be clearly distinguished from collision products. Stability of response and insensitivity to pile-up also promoted BCM to the preferred luminosity monitor of ATLAS. In addition six pad pCVD diamond sensors per side are hooked up to the LHC machine BLM system, replacing the ionization chambers as the current source. Current is monitored at $40 \mu$ s intervals, and the beams aborted if the threshold is exceeded in the same time bin in two out of six sensors on each side. The LHC beams were aborted twice in 2011 upon a clear signature of an "UFO" (dust speck) traversing the LHC beam near ATLAS.

The success of diamond-based detectors led ATLAS to plan the installation of a diamond based luminosity and beam spot monitor - DBM. This project is executed in the scope of the ATLAS Insertable B-Layer (IBL) project [12]. 24 pCVD sensors, $\sim 2 \mathrm{~cm} \times 2 \mathrm{~cm}$ in size, will be grouped by three in eight telescopes pointing to the interaction point from the forward direction ( $\mathrm{r} \sim 60 \mathrm{~mm}, \mathrm{z} \sim \pm 900 \mathrm{~mm})$. Sensors will be patterned into 26880 pixels $(50 \mu \mathrm{m} \times 250 \mu \mathrm{m})$ and read out with the FE-I4 pixel read out chip. When installed during the LHC shutdown in 2013/14 it will constitute the largest diamond tracking detector ever deployed in HEP.

\section{References}

[1] CERN/LHCC Reports 97-3, 98-20, 2000-011, 2000-015, 2001-002, 2002-010, 2003-063, 2005003, 2006-010, 2007-002, 2008-005, https://indico.cern.ch/materialDisplay.py?contribId=8\&sessionId=0\&materialId=slides\&confId=19 4183.

[2] S. Zhao, "Characterization of the Electrical Properties of Polycrystalline Diamond Films", Ph.D. Dissertation, Ohio State University (1994).

[3] Diamond Detectors Ltd, 16 Fleetsbridge Business Centre, Upton Road, Poole, Dorset, BH17 7AF, UK.

[4] Element Six Ltd, Kings Ride Park, Ascot, Berkshire, SL5 8BP, UK.

[5] II-VI INFRARED, 375 Saxonburg Blvd, Saxonburg, PA 16056, United States.

[6] P. Miyagawa, private communication, https://twiki.cern.ch/twiki/bin/viewauth/Atlas/RadiationBackgroundSimulations\#IBL_studies.

[7] W. de Boer, J. Bol, A. Furgeri, et al., "Radiation hardness of diamond and silicon sensors compared," Phys. Status Solidi A204(2007)3004-3010.

[8] Steffen Müller, "The Beam Condition Monitor 2 and the Radiation Environment of the CMS Detector at the LHC", PhD Thesis, University of Karlsruhe, CERN-THESIS-2011-085.

[9] M.Mikuž, V. Cindro, S. Cline et al., "Study of Polycrystalline and Single Crystal Diamond «Detectors Irradiated with Pions and Neutrons up to $3 \times 10^{15} \mathrm{~cm}^{-2 \alpha}, 2007$ IEEE Nuclear Science Symposium Conference Record, N44-5.

[10] N. Odell, "Measurements of the luminosity and normalised beam-induced background using the CMS Fast Beam Condition Monitor", these proceedings.

[11] V. Cindro, D. Dobos, I. Dolenc, et al., "The ATLAS Beam Conditions Monitor", JINST 3(2008)02004. 